\title{
A Note on Width-Parameterized SAT: an exact machine-model characterization
}

\author{
Periklis A. Papakonstantinou \\ University of Toronto
}

\begin{abstract}
We characterize the complexity of SAT instances with path-decompositions of width $w(n)$. Although pathwidth is the most restrictive among the studied width-parameterizations of SAT, the most time-efficient algorithms known for such SAT instances run in time $2^{\Omega(w(n))}$, even when the path-decomposition is given in the input. We wish to better understand the decision complexity of SAT instances of width $\omega(\log n)$. We provide an exact correspondence between $\mathrm{SAT}_{\mathrm{pw}}[w(n)]$, the problem of SAT instances with given path decomposition of width $w(n)$, and NL $[r(n)]$, the class of problems decided by logspace Turing Machines with at most $r(n)$ passes over the nondeterministic tape. In particular, we show that $\operatorname{SAT}_{\mathrm{pw}}[w(n)]$ is hard for $\mathrm{NL}\left[\frac{w(n)}{\log n}\right]$ under $\log$-space reductions. When $\operatorname{NL}\left[\frac{w(n)}{\log n}\right]$ is closed under logspace reductions, which is the case for the most interesting $w(n)$ 's, we show that $\operatorname{SAT}_{\mathrm{pw}}[w(n)]$ is also complete.
\end{abstract}

\section{Introduction}

A common way to consider subproblems of SAT is to parameterize the input according to some graph-theoretic width-parameter measured on a graph associated with the input formula. The incidence graph is a canonical example of such a graph. Treewidth, pathwidth, cliquewidth and so on, are width parameters for CNF instances, extensively studied both in theoretical and empirical research. This line of research gives algorithms (for inputs with restricted parameters) with running times significantly better than that of exhaustive search. Different parameterizations of SAT have been studied under different settings, but in general no algorithms faster than $2^{O(w(n))}$ are known, even when the width $w(n)$ corresponds to pathwidth, the most restrictive among the studied width-parameters. We observe that the Exponential Time Hypothesis (ETH), a strong complexity assumption, can be used to obtain lower bounds on the time complexity of SAT instances of pathwidth $\omega(\log n)$. One motivating question for this note is whether we can relate the non-existence of polytime algorithms for such SAT instances with complexity assumptions weaker than the ETH.

We denote by $\operatorname{SAT}_{\mathrm{pw}}[w(n)]$ the set of satisfiable CNFs with given path decompositions of width $w(n)$, where $n$ is the input length (definitions given later on in this section). By providing the path decomposition in the input in some sense we mod-out the difficulty of computing the pathwidth.

Conjecture. Let $w(n)=\omega(\log n)$. Then, $\operatorname{SAT}_{\mathrm{pw}}[w(n)] \notin \mathrm{P}$.

Conditioned on ETH this conjecture becomes true (see "Our results"). ETH [6] states that the $N$ variable 3SAT can't be solved in time $2^{o(N)}$. Making further progress seems difficult, partly because the precise complexity of $\operatorname{SAT}_{\mathrm{pw}}[w(n)]$ is unknown. In [4] it is observed that for $k$-CNFs with path decompositions of width $w(n)$ the $2^{O(w(n))}$ running time can be improved to nondeterministic space $O(w(n))$. This improvement is far from an exact characterization, since $\operatorname{NSPACE}(w(n))$ is believed to contain sets not in NP, if $w(n) \in \omega(\log n)$. In this note we use elementary techniques to precisely characterize the complexity of $\operatorname{SAT}_{\mathrm{pw}}[w(n)]$ by identifying a correspondence between two seemingly unrelated concepts.

Verifier Turing Machines. Let $M$ be a logspace Turing Machine equipped with a polynomially long, two-way nondeterministic tape (nd-tape). We call $M$ a Verifier Turing Machine, or vTM. Machines with one pass over the nd-tape characterize NL. On the other hand, polynomially many passes characterize NP, since NP predicates can be verified in logspace. We denote by $\mathrm{NL}[r(n)]$ the class of sets accepted by vTMs where the head of the nd-tape is 
allowed to reverse $r(n)-1$ times, i.e. making $r(n)$ passes. Thus, $\mathrm{NL}[1]=\mathrm{NL}$ and $\mathrm{NL}\left[n^{O(1)}\right]:=\cup_{c>0} \mathrm{NL}\left[n^{c}\right]=\mathrm{NP}$. Note that other than the nd-tape, all other tapes have an unbounded number of reversals, as in a standard logspace TM.

Understanding the power of vTMs is of independent interest. For example, for which $r(n)>1, \mathrm{NL}[r(n)] \subseteq \mathrm{P}$ ? We relate $\mathrm{NL}[\cdot]$ and $\mathrm{SAT}_{\mathrm{pw}}[\cdot]$ in a very strong sense.

Theorem 1. Let $w(n)$ be constructible in space $O(\log n)$. If $N L\left[\frac{w(n)}{\log n}\right]$ is closed under logspace reductions, then $S A T_{\mathrm{pw}}[w(n)]$ is complete for $N L\left[\frac{w(n)}{\log n}\right]$.

In "Our results" paragraph we give implications of our main theorem. For example, based on the interplay between $\mathrm{SAT}_{\mathrm{pw}}$ and vTMs we obtain lower bounds on the number of nd-tape head reversals for vTMs.

Remark 2. Several details matter for the exact characterization to work. For instance, it is crucial that the parameter is the number of passes over the nd-tape and not some other measure of "use" of nondeterminism. Furthermore, the same characterization wouldn't work for treewidth, since SAT $\mathrm{pw}_{\mathrm{pw}}[\log n]$ is complete for NL, but we conjecture that the same problem for treewidth is complete for LOGCFL (one can show containment [4]), and it is thought that NL $\subsetneq$ LOGCFL.

Related work. Width-parameterizations of SAT are studied as a case of parameterized complexity (cf. [3]). There is an extensive body of work, too large to be cited here (e.g. see [8] for an older survey). Regarding our motivating conjecture there are some very interesting works for similar questions, in particular for treewidth parameterized CSPs and for CLIQUE $[2,5,7]$.

Definitions and notation. Let $\phi$ be a CNF formula with variables $L:=\left\{x_{1}, \ldots, x_{N}\right\}$ and clauses $R:=\left\{C_{1}, \ldots, C_{m}\right\}$. The incidence graph of $\phi$ is a bipartite graph with bipartization $(L, R)$, and there is an edge between a variable-vertex $x_{i} \in L$ and a clause-vertex $C_{j} \in R$, iff $x_{i}$ appears (signed) in $C_{j}$. Path and tree decompositions are defined over arbitrary graphs as follows. A tree decomposition of a graph $G=(V, E)$ is the pair $\tau:=\left(\left\{X_{i}: i \in I\right\}, T=(I, F)\right)$, such that $X_{i} \subseteq V, \cup X_{i}=V, T$ is a tree such that: (i) for every $\{u, v\} \in E$ there exists $i \in I$ such that $u, v \in X_{i}$, (ii) for every $u \in V$ let $X_{i_{1}}, \ldots, X_{i_{k}}$ be the collection of all sets containing $u$, then the subgraph of $T$ induced by $i_{1}, \ldots, i_{k} \in I$ is connected (i.e. it is a tree). The treewidth of $G$ is the minimum width over all decompositions, where the width of a decomposition is $\max _{i}\left|X_{i}\right|-1$. By restricting the tree $T$ to a path we define the path decomposition, and the pathwidth of $G$. It is well-known that for a graph $G, \operatorname{tw}(G) \leq \operatorname{pw}(G) \leq(\log |V|) \operatorname{tw}(G)$. Finally, we consider ordered $k$-CNFs $\Phi$ each being a sequence of clauses each consisting of at most $k$ literals. For the given ordering the distance of a variable $x$ is the index of the clause of the last appearance of $x$ minus the first appearance of $x$. The diameter of $\Phi, \operatorname{diam}(\Phi)$ is the maximum distance over its variables. The relevant computational problems are given below.

Notation. All logarithms are of base 2. For real-valued parameters, where integer parameters are assumed, we implicitly consider their ceilings, rounding up to the closest integer. All reductions are many-to-one and logspace. We denote by $n$ the length of the input, by $N$ the number of variables in a CNF and by $M$ the number of clauses. All widths are functions of $n$. We only consider non-decreasing functions $w, r: \mathbb{Z}^{+} \rightarrow \mathbb{Z}^{+}$constructible in logarithmic space.

$$
\mathrm{SAT}_{\mathrm{pw}}[\mathbf{w}(\mathbf{n})]
$$

Input: A CNF formula $\phi$ together with a path decomposition of the incidence graph of width $\leq w(n)$

Question: Is $\phi$ satisfiable?

$$
\begin{aligned}
& k \text {-SAT }_{\text {diam }}[\mathbf{w}(\mathbf{n})] \\
& \text { Input: An ordered CNF formula } \Phi \text { where } \\
& \operatorname{diam}(\Phi) \leq w(n) \\
& \text { Question: Is } \Phi \text { satisfiable? }
\end{aligned}
$$

These problems are inter-reducible within logspace (see Lemma 3 below). Usually, we consider constant clause size CNFs for $\mathrm{SAT}_{\text {diam }}$, and arbitrary CNFs for our central problem $\mathrm{SAT}_{\mathrm{pw}}$.

Our results. Our main result is the completeness part of Theorem 1. In [4] it is shown that $3-\mathrm{SAT} \mathrm{Tw}_{\mathrm{pw}}[w(n)] \in$ $\operatorname{NSPACE}(w(n))$, and that $3-\mathrm{SAT}_{\mathrm{pw}}[w(n)]$ and 3-SAT $\mathrm{T}_{\text {diam }}[w(n)]$ are logspace reducible to each other. Thus, the results of the next section imply Lemma 3 and Corollary 4 . In particular, Theorem 1 and Corollary 4 are proved in Section 2. Lemma 3 directly follows by the foregoing results from [4], Lemma 11, and by padding. Here, by padding we mean that we extend a propositional formula by adding new clauses each of which contains a single, fresh variable. 
Lemma 3. $S A T_{\text {diam }}[w(n)], S A T_{\mathrm{pw}}[w(n)], 3-S A T_{\mathrm{pw}}[w(n)]$, and $S A T_{\mathrm{pw}}[c w(n)], c>0$ are inter-reducible.

Corollary 4. (i) $N L[r(n)]=N L[c r(n)], c>0$, and (ii) $N L[r(n)] \subseteq N S P A C E(r(n) \log n)$.

Lemma 3 is somewhat interesting since there is a family of 3-CNFs where every variable appears only a constant number of times, the formulas are of pathwidth $O(\log n)$ and every ordering of the clauses has diameter $\Omega\left(\frac{n}{\log n}\right)$ [4]. This combinatorial exponential gap is being smoothed out through logspace transformations.

As mentioned, the most interesting cases for $w(n)$ are those where $\mathrm{NL}[w(n) / \log n]$ is closed under $\operatorname{logspace}$ reductions; e.g. NL[1], NL[ $\log \log n], \mathrm{NL}\left[\log ^{k} n\right]$. The closure under $\operatorname{logspace}$ reductions is immediate by Corollary 4 , which also implies that a constant number of passes over the nd-tape can't get us outside NL; i.e. $\mathrm{NL}[O(1)]:=$ $\cup_{i=1}^{\infty} \mathrm{NL}[i]=\mathrm{NL}$.

Since $\mathrm{NL}[O(1)]=\mathrm{NL}$ and $\mathrm{NL}\left[n^{O(1)}\right]=\mathrm{NP}$, it is natural to ask what is the smallest $r(n)$ such that $\mathrm{NL}[r(n)] \supseteq \mathrm{P}$. Under a widely believed complexity assumption, Corollary 4 implies that it is not possible to capture $\mathrm{P}$ before NP.

Corollary 5. There does not exist $k>0$ such that $N L\left[n^{k}\right] \supseteq P$, unless $P \subseteq D \operatorname{SPACE}\left(n^{k^{\prime}}\right)$, for some $k^{\prime} \in \mathbb{Z}^{+}$.

It follows by Corollary 4 and Theorem 1 that there are $r(n)$ 's where $\mathrm{NL}[r(n)]$ is not closed under logspace reductions, modulo complexity assumptions. For example, if $\mathrm{NL}\left[n^{\epsilon}\right]$ is closed under log-space reductions then $\mathrm{NP} \subseteq \mathrm{NL}\left[n^{\epsilon}\right] \subseteq \mathrm{DSPACE}\left(n^{\epsilon^{\prime}}\right)$

A perhaps surprising consequence of the relation between $\mathrm{SAT}_{\mathrm{pw}}[\cdot]$ and $\mathrm{NL}[\cdot]$ is Corollary 7 , which follows by Lemma 8 and the following lemma. Recall that ETH states that the $N$-variable 3 -SAT cannot be solved in time $2^{o(N)}$.

Lemma 6. Let $w(n)=\omega(\log n)$. If ETH holds then $3-S A T_{\mathrm{pw}}[w(n)] \notin P$.

Proof. Say that $w(n) \in \omega(\log n)$, and 3-SAT $\mathrm{pw}_{\mathrm{pw}}[w(n)] \in \mathrm{P}$. Let $\Phi$ be a 3-CNF with $M=O\left(\frac{n}{\log n}\right)$ clauses and $N$ variables. By a padding argument we can decide $\Phi$ in time $2^{o(M)}$, and by the Sparsification Lemma [6], this implies an $2^{o(N)}$ algorithm, contradicting ETH.

Corollary 7. Let $r(n)=\omega(1)$. If ETH holds then there exists $L \in N L[r(n)]$, such that $L \notin P$.

An example of an $L$ in Corollary 7 is 3-SAT $\mathrm{pw}_{\mathrm{pw}}[\omega(\log n)]$ (note that Lemma 8 also holds for 3-SAT $\mathrm{pw}[w(n)]$ ).

\section{Proof of Main Theorem}

Lemma 3 states that $3-\mathrm{SAT}_{\text {diam }}$ and $\mathrm{SAT}_{\mathrm{pw}}$ are interreducible, and thus Lemmas 8 and 9 imply Theorem 1. Lemma 11 allows us to restrict to 3 -CNFs, and thus to conclude Lemma 3.

Lemma 8. $3-S A T_{\text {diam }}[w(n)] \in N L\left[\frac{w(n)}{\log n}\right]$, for $w(n)=\Omega(\log n)$.

Proof. Let $\Phi$ be an ordered 3-CNF formula of size $n$ and diameter $\leq w(n)$. we now present a nondeterministic logspace algorithm that in each pass over the nondeterministic tape verifies that $\frac{n}{w(n)} \log n$ new clauses are satisfied. Hence, $\Phi$ is covered in $\leq \frac{w(n)}{\log n}$ passes.

Consider an ordering of the variables of (the clause-ordered) $\Phi$ consistent with the ordering of the clauses. We view the nd-tape as containing a truth assignment, where the $i$-th bit of the tape is the truth value of the $i$-th variable. By definition of diameter, if the $j$-th clause in the input has a variable which corresponds to the $i$-th bit on the ndtape, then the $(j+w(n))$-th clause contains variables with index $\geq i$. For simplicity of presentation suppose that all divisions are for divisible integers. Partition the ordered clauses into consecutive blocks each containing $\log n$ clauses. The algorithm is iterative, and in each iteration it deals with exactly one such block which we call "effective block". Reserve space for a $\log n$ bit vector on the worktape. Each bit of this vector is associated with a clause in the "effective block". That is, the input tape is partitioned in blocks $b_{1}, b_{2}, \ldots, b_{n / \log n}$. We define the distance of two blocks to be the number of clauses between them. Two blocks of distance at least $w(n)$ do not share any variables. Partition the input tape further into super-blocks, each of size $w(n)$. That is, we have $\frac{n}{w(n)}$ super-blocks each containing $\alpha:=\frac{w(n)}{\log n}$ blocks. That is, the blocks can be re-indexed as follows: $b_{1,1}, b_{1,2}, \ldots, b_{1, \alpha}, b_{2,1}, \ldots, b_{2, \alpha}, \ldots, b_{n / w(n), \alpha}$. Iteratively make passes over the nd-tape, where in each pass $\frac{n}{w(n)}$ blocks are verified to be satisfiable, as follows. 
In the odd numbered passes the nondeterministic head scans from the left to the right end of the nd-tape, and symmetrically for the even numbered ones. We present the algorithm for an odd numbered iteration; symmetrically the algorithm works for the even numbered one. In the $i$-th iteration we verify the satisfiability of $b_{j, i}$ 's, $j=1,2, \ldots, \frac{n}{w(n)}$. Start moving the head on the nd-tape from left to right until we encounter the first variable $x$ that appears in $b_{1, i}$. Check which (if any) clauses of $b_{1, i}$ are satisfied by $x$ and record this on the corresponding bits on the reserved $\log n$ bit vector on the worktape. Continue this way until the last variable in the block is read. If the reserved space shows that there is a clause not satisfied then reject. Else, the nondeterministic head has not read yet any variable from $b_{2, i}$. Move the nondeterministic head further and repeat for $b_{2, i}$ as for $b_{1, i}$; similarly, for $b_{3, i}, \ldots, b_{n / w(n), i}$.

This algorithm is well-defined since the truth values for the verification they are located at most $w(n)$ to the left and $w(n)$ to the right of each block. The correctness of this logspace algorithm is transparent in its description.

An easy modification of the above algorithm makes the lemma true for $3-\mathrm{SAT}_{\mathrm{pw}}[w(n)]$.

Lemma 9. 3-SAT $T_{\text {diam }}[w(n)]$ is hard for $N L\left[\frac{w(n)}{\log n}\right], w(n)=\Omega(\log n)$.

Proof. Let $r(n)=\frac{w(n)}{\log n}$. Since $w(n)$ is computable in space $O(\log n), r(n)$ is also $\operatorname{logspace}$ computable. Fix arbitrary $L \in \mathrm{NL}[r(n)]$ and let $M$ be a 1-tape logspace vTM accepting $L$, which makes $r(n)$ passes over its nd-tape, and is standardized as follows.

(i) $M$ has a unique accepting configuration, (ii) $M$ is oblivious in the input head move, (iii) $M$ is oblivious in the nondeterministic head move and in fact it moves the nondeterministic head in each step in a left to right end (and right to left end) fashion. We can easily achieve (i), (ii) and make the nondeterministic head move oblivious by slowing down the computation. In particular, it is easy to make the nondeterministic head stay-put for a polynomial number of steps $p(n)$ in between moves, and make it move from the left to the right end of the polynomially $q(n)$-long tape and back. To standardize the machine to satisfy (iii) we proceed as follows. Construct a nondeterministic logspace TM verifying in a single pass whether its nd-tape is of the form $\underbrace{b_{1} b_{1} \ldots b_{1}}_{p(n) \text { times }} \underbrace{b_{2} b_{2} \ldots b_{2}}_{p(n) \text { times }} \ldots \underbrace{b_{q(n)} b_{q(n)} \ldots b_{q(n)}}_{p(n) \text { times }}$ and reject if it is not. Composing this logspace machine with the previously standardized logspace machine we obtain a vTM $M$ for $L$, satisfying properties (i), (ii), and (iii). On an input of length $n$, our standardize TM makes polynomially many $t(n)$ steps.

The hardness follows by modifying the construction in Cook's theorem for the NP-completeness of SAT (cf. [1] pp. 48-50). For simplicity, we initially deal only with the case where the number of passes $r(n)=1$.

Case $r(n)=1$ : Let $x$ be an input of length $n$. We wish to construct an ordered 3-CNF formula $\Phi$ of diameter $O(\log n)$ (which by padding is sufficient), such that $\Phi$ is satisfiable iff $M$ accepts $x$. We define a configuration $\gamma$ to be a string which is the concatenation of the strings: (i) the $O(\log n)$ symbols of the worktape, encoding the worktape symbol which the head is on by a special symbol, (ii) the state of $M$, and (iii) the value of the nondeterministic bit at the current position in the nd-tape.

For our (standardized) vTM we wish to check whether there exists a sequence of configurations $\gamma_{1} \vdash \gamma_{2} \vdash \ldots \vdash$ $\gamma_{t(n)}$, where $\gamma_{1}$ is the initial configuration on $x$, and $\gamma_{t(n)}$ is the accepting configuration. Our formula will have variables $y_{i, j, \alpha}$; we will enforce the constraints that $y_{i, j, \alpha}=1$ iff symbol at position $j$ of the configuration at step $i$ is $\alpha$. We first express in the usual way that $\gamma_{1}$ is the initial configuration. The main technical part is to enforce for $i=1, \ldots, t(n)-1$ that $\gamma_{i} \vdash \gamma_{i+1}$. We can do this in the usual way, noting that the $j$-th symbol of $\gamma_{i+1}$ only depends on a constant number of symbols of $\gamma_{i}$, since (by obliviousness) the position read by the input head is determined by $i$. It is also important to realize that we do not need to encode any constraint on the bit read on the nd-tape since a new bit is read at each step, and since $r(n)=1$ no bit is ever reread. Lastly, we express in the usual way that $\gamma_{t(n)}$ is the accepting configuration. To see that our ordered formula is of diameter $O(\log n)$, the main point is that the subformula needed to express $\gamma_{i} \vdash \gamma_{i+1}$ has $O(\log n) 3$-literal clauses and uses only those variables associated with $\gamma_{i}$ and $\gamma_{i+1}$; diameter $O(\log n)$ comes from the fact that we encode both $\gamma_{i} \vdash \gamma_{i+1}$ and $\gamma_{i+1} \vdash \gamma_{i+2}$.

Case arbitrary $r(n)$ : If we perform the above reduction exactly as stated for $r(n)>1$ we do not have a valid reduction, since we must enforce consistency between the different time steps in which the same nondeterministic bit is visited. If we modify the above construction such that we reserve the same variable name for the same nondeterministic 
bit, then this results in a formula of diameter $n^{\Omega(1)}$. Instead, we will always use the same variable name for the $k$-th nondeterministic bit, and we will write together all the subformulas describing those $r(n)$ transitions where the nondeterministic head moves between the $k$-th and $(k+1)$-th position (in either direction). We do this for $k=$ $1, \ldots, z(n)$ each time creating a subformula of diameter $O(r(n) \log n)$, where $z(n)$ is the polynomial length of the nd-tape. This can also be expressed in a diagram, where we reorder the configurations denoting by $\gamma_{\text {pass,symbol }}$ the configuration when the nondeterministic head does the number pass scan over the nd-tape and it currently is at position symbol. Then, the computation starts by $\gamma_{1,1} \vdash \gamma_{1,2} \vdash \ldots \vdash \gamma_{1, z(n)}$ and the next configuration is $\gamma_{2, z(n)}$ since in the second pass the nondeterministic head moves from the right to the left end.

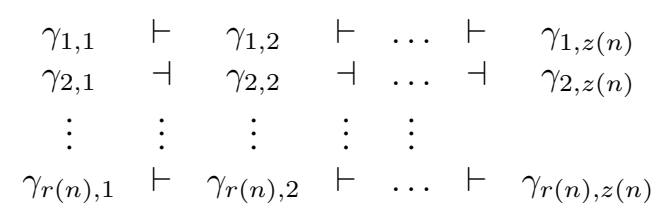

Matrix describing the computation

$$
\left(\begin{array}{c}
\gamma_{1, k} \\
\gamma_{2, k+1} \\
\vdots \\
\gamma_{r(n), k}
\end{array}\right) \vdash\left(\begin{array}{c}
\gamma_{1, k+1} \\
\gamma_{2, k} \\
\vdots \\
\gamma_{r(n), k+1}
\end{array}\right)
$$

Configurations regarding the nondeterministic bits $k$ and $k+1$

Remark 10. (i) Lemma 9 together with the algorithm [4] that decides 3-SAT $\operatorname{diam}[w(n)]$ in $\operatorname{NSPACE}(w(n))$ for every language in $\mathrm{NL}[w(n)]$ implies a nondeterministic $O(w(n) \log n)$ space algorithm. (ii) Standardizing the machine in the proof of Lemma 9 significantly simplifies the argument. An alternative way to proceed without this standardization involves renaming variables (e.g. by formulas of the form $x \leftrightarrow y$ ) and interpolating them in appropriate places (which is a non-obvious task).

Lemma 11. $S A T_{\mathrm{pw}}[w(n)]$ is reducible to $3-S A T_{\mathrm{pw}}[w(n)]$.

Proof. Fix 3-CNF $\Phi$ of size $n$, and fix a path decomposition $=\left\langle X_{1}, X_{2}, \ldots, X_{\alpha}\right\rangle$ of width $\leq w(n)$. Let $C=$ $\left\{l_{1}, \ldots, l_{k}\right\}$ be a clause of $\Phi$, and assume that $l_{1}, \ldots, l_{k}$ respects the ordering of the variables in $P$. We introduce new variables $y_{1}, \ldots, y_{k-1}$, and note that a truth assignment to the old variables satisfies $C$ iff some extension to the new variables satisfies all $\left\{l_{1}, \neg y_{1}\right\},\left\{y_{1}, l_{2}, \neg y_{2}\right\}, \ldots,\left\{y_{k-2}, l_{k-1}, \neg y_{k-1}\right\},\left\{y_{k-1}, l_{k}\right\}$.

Let $X_{m}$ contain clause $C$, and say that the literals $l_{i+1}, \ldots, l_{i+k_{m}}$ are the literals of $C$ whose variables are in $X_{m}$ and they are not in an earlier $X_{m^{\prime}}$ that contains $C$. We then replace $X_{m}$ by the subpath $X_{m}^{1}, \ldots, X_{m}^{k_{m}}$ where $X_{m}^{j}$ is obtained from $X_{m}$ by removing $C$, adding the new variables from the new clause containing $l_{i+j}$, and adding that new clause as well. This log-space transformation results in a valid path decomposition of width $\leq 2 w(n)$. The resulted formula is satisfiable iff $\Phi$ is satisfiable, and the theorem follows by padding.

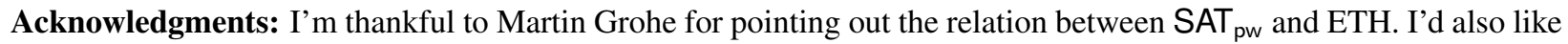
to thank Toniann Pitassi for the helpful discussions, and Charles Rackoff for the close attention to my work.

\section{References}

[1] Ding-Zhu Du and Ker-I Ko. Theory of Computational Complexity. John Wiley and Sons, 2000.

[2] U. Feige and J. Kilian. On limited versus polynomial nondeterminism. Chicago J. Theor. Comp. Sci., 1997.

[3] J. Flum and M. Grohe. Parameterized complexity theory. Springer-Verlag, Berlin, 2006.

[4] K. Georgiou and P. A. Papakonstantinou. Complexity and algorithms for well-structured k-SAT instances. In SAT'08, pages 105-118, 2008.

[5] M. Grohe. The complexity of homomorphism and constraint satisfaction problems seen from the other side. J. ACM, 54(1):(electronic), 2007 (also FOCS'03). 
[6] R. Impagliazzo, R. Paturi, and F. Zane. Which problems have strongly exponential complexity? J. Comp. Sys. Sci., 63(4):512-530, 2001 (also FOCS'98).

[7] D. Marx. Can you beat treewidth? In FOCS'07, pages 169-179, 2007.

[8] S. Szeider. On fixed-parameter tractable parameterizations of SAT. In SAT’03, pages 188-202, 2003. 